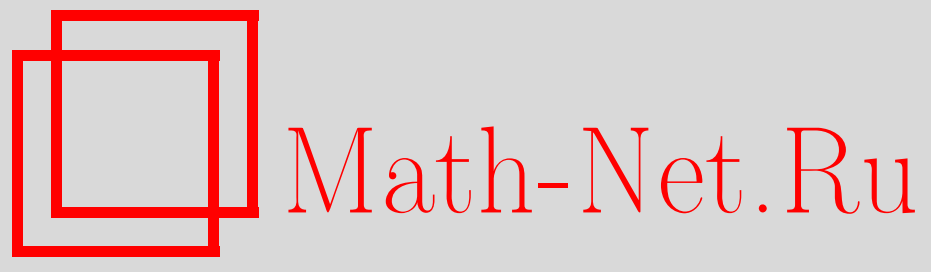

Ю. А. Неретин, С. М. Хорошкин, Математические работы Д. П. Желобенко, УМН, 2009, том 64, выпуск 1, 178 188

DOI: https://doi.org/10.4213/rm9279

Использование Общероссийского математического портала Math-Net.Ru подразумевает, что вы прочитали и согласны с пользовательским соглашением http://www . mathnet.ru/rus/agreement

Параметры загрузки:

IP: 34.229 .45 .116

26 апреля 2023 г., $17: 28: 38$

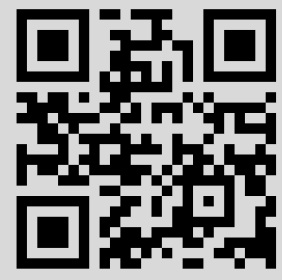




\section{Математические работы Д. П. Желобенко}

1. Краткие биографические сведения. Основные работы Дмитрия Петровича Желобенко (Ульяновск, 1934 - Москва, 2006) посвящены теории представлений полупростых групп и алгебр Ли, а также некоммутативному гармоническому анализу.

Он окончил физический факультет МГУ (1958) и аспирантуру Математического института им. В. А. Стеклова под руководством С. В. Фомина и М. А. Наймарка (1961). Защитил кандидатскую диссертацию "Гармонический анализ функций на группе Лоренца и некоторые вопросы теории линейных представлений" в 1962 г. Докторская диссертация "Гармонический анализ функций на полупростых группах Ли и его приложения к теории представлений" была защищена в МИАНе в 1972 г. (оппоненты И. М. Гельфанд, И. Р. Шафаревич, А. А. Кириллов). В 1974 г. был докладчиком на Международном математическом конгрессе в Ванкувере ${ }^{1}$.

Начиная с 1961 г. и до смерти, работал в Университете Дружбы Народов им. П. Лумумбы ${ }^{2}$. По-видимому, данная работа давала поле для реализации его преподавательских способностей, но возможностей найти аспирантов у него было не много ${ }^{3}$.

В 1963-1978 гг. в институте им. В. А. Стеклова проходил семинар М. А. Наймарка, Д. П. Желобенко и А. И. Штерна по теории представлений. Среди докладчиков были, например, Ф. А. Березин, Н. Я. Виленкин, Р. С. Исмагилов, А. А. Кириллов, Э. В. Киссин, Г.Л. Литвинов, В.И. Манько, М.Б. Менский, В.Ф. Молчанов, М.Г. Крейн, Г. И. Ольшанский, И. Сигал (Irving Segal), В.Н. Толстой, Ч. Фояш (Ciprian Foiaş), Дж. Хаджиев, П. Халмош (Paul Halmos), А. Я. Хелемский, Э. Хьюит (Edwin Hewitt). Дмитрий Петрович многократно читал лекции для физиков, в частности в Дубне (1965), в Матфизическом колледже Московского независимого университета в начале 1990-х, в Софийском университете (1973-74), а также на ряде физических конференций.

Начиная с 1959 г. и до последних дней жизни Дмитрий Петрович был серьезно болен (в результате неудачного горного похода ${ }^{4}$ ).

Дмитрий Петрович - автор 9 книг ${ }^{5}$ и более 70 статей (согласно MathSciNet и Zentralblatt). Цель нашего краткого обзора - обсудить те из его работ, которые кажутся

\footnotetext{
${ }^{1}$ Фактически за границу его тогда “не выпустили". Кстати, доклад М. А. Наймарка на конгрессе в Ницце в 1970 г. по содержанию был совместным с Д. П. Желобенко.

${ }^{2}$ УДН; позднее переименован в Российский университет дружбы народов (РУДН).

${ }^{3}$ При этом ему иногда приходилось сталкиваться с административными препонами при попытке "взять" способного студента. Вот список аспирантов, защитивших диссертацию под руководством Д.П. Желобенко, взятый нами из Curriculum Vitae: М. С. Аль-Натор, А. С. Казаров, А. Г. Князев, А. В. Луцюк. Учеником Желобенко отчасти был Виджай Джха, впоследствии работавший в теории чисел.

${ }^{4}$ Желобенко вывел туристическую группу на Алтае, когда та оказалась в критическом положении. Вот отрывок из письма В.И. Чилина: "Из бесед с Дмитрием Петровичем я узнал, что он заболел после тяжелого горного похода. Разыскивая дорогу, он по договоренности с руководителем группы ушел вперед, затем начался сильный снегопад, и он не смог в наступающих сумерках вернуться к месту ночлега. И ему пришлось всю ночь двигаться по кругу, чтобы не замерзнуть. В результате он сильно простудился, что и привело к серьезной болезни".

${ }^{5}$ Две из них, [57] и [58], сейчас находятся в печати.
} 
нам наиболее значительными. По понятным причинам этот обзор не полон. Кроме того, поставив себе такую цель, мы уже не можем выйти за рамки чисто профессионального текста.

Издательство МЦНМО и Ю. Н. Торхов сейчас готовят издание новой книги Желобенко "Гауссовы алгебры". Предполагается включить туда воспоминания его коллег и друзей, Г. Л. Литвинова и В. Ф. Молчанова, а также учеников, М. С. Аль-Натора, И. В. Гольдеса, А. С. Казарова, В. Р. Нигматуллина. Там же должна быть библиография его статей.

2. Книга "Компактные группы Ли и их представления" [47] (1970). Это классическая книга, которая и по сей день остается одной из лучших книг по теории представлений, причем как в жанре учебника (или вводной книги), так и в жанре монографии. Этот очень своеобразный как по структуре, так и по стилю текст ${ }^{6}$ в итоге оказался образцом "математики с человеческим лицом". Он был рассчитан на читателя с подготовкой в 2-3 курса тогдашних математических (физических) факультетов, он является и книгой для начального ознакомления или обучения, и книгой, интересной для специалистов, и справочником в определенной области.

Мы не обсуждаем ее содержания (лучше почитать саму книгу) и лишь отметим, что ее "сложные главы" (задачи спектрального анализа) во многом основаны на работах самого Д. П. Желобенко, в частности на [39], [40] ${ }^{7}$.

Оригинальной находкой Д. П. Желобенко является последовательное изложение большого числа классических задач теории представлений в рамках так называемого метода $Z$-инвариантов. Принцип метода $Z$-инвариантов состоит в реализации представлений полупростых групп в виде функций на максимальной унипотентной (строго треугольной) подгруппе, удовлетворяющих определенной системе дифференциальных уравнений [39], [40]. В сущности, этот метод предвосхищает теорию $D$-модулей на флаговых пространствах ${ }^{8}$ (см. работы А. Бейлинсона и И. Бернштейна [4], Ж.-Л. Брылинского и М. Кашивары [10].

3. Книга "Гармонический анализ на комплексных полупростых группах Ли" [50] (1974). В своей основе это оригинальная работа, основанная на статьях Д. П. Желобенко ${ }^{9}$ [41]-[46], [48], [49], [59], [60] 1963-1973 гг., частично совместных с М. А. Наймарком. Основной результат - теорема Желобенко-Наймарка о классификации всех неприводимых представлений ${ }^{10}$ комплексных полупростых групп Ли. Напомним это утверждение для случая группы $G=\mathrm{GL}(n, \mathbb{C}){ }^{11}$

Через $\mathrm{U}(n)$ мы обозначим подгруппу всех унитарных матриц. Обозначим через $T \subset G$ подгруппу верхнетреугольных матриц. Для $A \in T$ обозначим через $a_{j j}$ ее диагональные элементы. Фиксируем набор комплексных чисел $p_{1}, \ldots, p_{n}, q_{1}, \ldots, q_{n}$. Рассмотрим характер $T \rightarrow \mathbb{C}^{*}$, заданный формулой

$$
\chi_{p, q}(A)=\prod_{j=1}^{n} a_{j j}^{p_{j}} \bar{a}_{j j}^{q_{j}} .
$$

Чтобы комплексные степени имели смысл, нужно, чтобы все $\left(p_{j}-q_{j}\right)$ были целыми.

\footnotetext{
${ }^{6}$ Кажется, самого Дмитрия Петровича до конца его жизни удивляла очевидная популярность его "неправильно" (“для физиков") написанной книги.

${ }^{7}$ Стоит отметить, что на эти статьи большое влияние оказала работа Р. Годемана [21].

${ }^{8}$ Строго треугольная подгруппа является “большой клеткой” флагового пространства.

${ }^{9}$ Некоторые из них пересекаются со статьями М. Дюфло того же времени, см. ссылки в [14], [50].

10 в пространствах Фреше с точностью до инфинитезимальной эквивалентности модулей $K$-конечных векторов.

${ }^{11}$ Ниже также $G$ - произвольная полупростая группа, $K$ - ее максимальная компактная подгруппа, $\mathfrak{g} \supset \mathfrak{k}-$ их алгебры Ли. Сейчас $G=\mathrm{GL}(n, \mathbb{C}), K=U(n)$.
} 
Рассмотрим представление $\rho_{p, q}$ группы $G$, индуцированное с представления $\chi_{p, q}$ группы $T .^{12}$ То, что получилось, называется представлением основной серии. Для $p, q$ общего положения ${ }^{13}$ представления основной серии неприводимы. При исключительных значениях параметра представление $\rho_{p, q}$ имеет конечный композиционный ряд Жордана-Гёльдера. Далее, при $p, q$ общего положения одновременная перестановка наборов $\left(p_{1}, \ldots, p_{n}\right)$ и $\left(q_{1}, \ldots, q_{n}\right)$ приводит к эквивалентному представлению. ${ }^{14}$ Для исключительных значений $p, q$ такие представления не эквивалентны, но композиционные ряды имеют одинаковые факторы (ниже мы используем слово “подфактор").

Теперь сформулируем теорему Желобенко-Наймарка. Неприводимые представления $\mathrm{GL}(n, \mathbb{C})$ находятся во взаимно однозначном соответствии с орбитами симметрической группы $S_{n}$ на множестве наборов $\left(p_{1}, \ldots, p_{n} ; q_{1}, \ldots, q_{n}\right)$. А именно, для каждой орбиты берется один из подфакторов соответствующего $\rho_{p, q}$. Опишем подфактор более точно. Без ограничения общности можно положить $p_{1}-q_{1} \geqslant p_{2}-q_{2} \geqslant$ $\cdots \geqslant p_{n}-q_{n}$, теперь надо взять тот подфактор (он является подпредставлением), чье ограничение на подгруппу $K=\mathrm{U}(n)$ содержит представление со старшим весом $\left(p_{1}-q_{1}, \ldots, p_{n}-q_{n}\right)$.

Сама эта теорема (1966) являлась частью 30-летней истории вокруг теоремы Хариш-Чандры о подфакторе. Основные серии и параболическое индуцирование были введены в книге И. М. Гельфанда и М. А. Наймарка [18]. Хариш-Чандра в 1954 г. [22] доказал, что любое неприводимое представление полупростой группы Ли реализуемо как подфактор основной серии ${ }^{15}$. Ф. А. Березин немедленно (1956) [5], [6] попытался превратить этот результат в явную классификацию в случае комплексных групп. ${ }^{16}$ Десять лет спустя (1966) была получена теорема Желобенко-Наймарка, затем в 1973 г. Р. Ленглендс [27] получил классификацию всех неприводимых представлений вещественных групп, а В. Касселман [11] в 1975 г. получил теорему о подпредставлении. ${ }^{17,} 18$

\footnotetext{
12 Для читателя, привычного к другому языку: мы рассматриваем представления $\mathrm{GL}(n, \mathbb{C})$ в гладких сечениях комплексных линейных расслоений над флаговым пространством.

13 Условие исключительности $p_{i}-p_{j} \in \mathbb{Z}, q_{i}-q_{j} \in \mathbb{Z}$ для хотя бы одной пары $i, j$.

14 Для читателя чуть-чуть знакомого с группой Лоренца $\operatorname{SL}(2, \mathbb{C})$ и теорией представлений. Это почти очевидно. Во-первых, для $\mathrm{GL}(2, \mathbb{C})$ это общеизвестный и легко проверяемый факт. Далее, обозначим через $T_{i}$ обобщенную верхнетреугольную подгруппу, где элементу $a_{(i+1) i}$ группы, стоящему ниже диагонали, разрешено быть ненулевым. Группа Ли $T_{i}$ имеет $\mathrm{SL}(2, \mathbb{C})$ в качестве полупростого множителя. Теперь наше представление можно получить последовательным индуцированием с $T$ на $T_{i}$, а далее на $G$. Но теперь для $\mathrm{SL}(2, \mathbb{C})$ мы можем переставить параметры $\left(p_{i}, q_{i}\right)$ и $\left(p_{i+1}, q_{i+1}\right)$.

${ }^{15}$ см. также [13; раздел 9.4].

16 До классификации в буквальном смысле слова статья все же "не дотягивала", точнее, окончательная классификационная теорема была сформулирована без доказательства. В этой работе были обнаружены серьезные пробелы, вслед за этим последовала дискуссия между Хариш-Чандрой и Березиным по поводу правильности этой статьи. Березин в следующей статье ("Письмо в редакцию") заполнил эти пробелы. Подход Березина (с доведением до классификации) изложен в Добавлении А к обсуждаемой книге Желобенко (но надо отметить, что Желобенко применяет дополнительные соображения). Кстати, в обсуждаемой работе Березина были посчитаны радиальные части операторов Лапласа на полупростых группах, что, возможно, было не менее важно, чем начавшаяся “борьба" за классификацию представлений.

17 Любое неприводимое представление - подпредставление в основной серии.

18 Доказательства были опубликованы в книге А. Бореля и Н. Валлаха [8] (1980) и статье В. Касселмана и Д. Миличича [12] (1982); см. также книгу А. Кнаппа [25]. Вещественный случай принципиально сложнее комплексного, но и результаты менее прозрачны. Классификация всех представлений опирается на классификацию дискретных серий. Сами по себе представления дискретных серий - сложные объекты, известно несколько их описаний, но все они далеки от прозрачности. После появления работы М. Фленстеда-Йенсена [17] казалось, что дискретные серии вот-вот будут вполне поняты. Тридцать лет спустя работа Фленстед-Йенсена сама остается непонятой.
} 
Желобенко [44] также вводит следующую структуру. ${ }^{19}$ Обозначим через $U(\mathfrak{g})$ обертывающую алгебру алгебры Ли $\mathfrak{g}$, а через $U(\mathfrak{k})$ обертывающую алгебру подалгебры k. Пусть $\pi_{\lambda}$ обозначает неприводимые представления группы $K$, через $\lambda$ обозначен старший вес. Через $\operatorname{ker} \pi_{\lambda} \subset U(\mathfrak{k})$ мы обозначим двусторонний идеал, состоящий из элементов $v$ таких, что $\pi_{\lambda}(v)=0$. Через $U_{\lambda \mu}$ мы обозначим множество всех $u \in U(\mathfrak{g})$ таких, что

$$
\left(\operatorname{ker} \pi_{\lambda}\right) u \subset U(\mathfrak{g})\left(\operatorname{ker} \pi_{\mu}\right) .
$$

Легко проверить, что $U_{\lambda \mu} U_{\mu \nu} \subset U_{\lambda \nu}$. Поэтому мы получаем категорию, объектами которой являются доминантные веса $\lambda$, а морфизмами - элементы $u \in U_{\lambda \mu}$.

Для представления группы $G$ в пространстве $H$ рассмотрим его ограничение на подгруппу $K$, оно разлагается в прямую сумму $H=\bigoplus H_{\lambda}$, где в $H_{\lambda}$ действует представление $K$, кратное $\pi_{\lambda}$. Легко проверить, что $u \in U_{\lambda \mu}$ переводит $H_{\mu}$ в $H_{\lambda}$, т. е. мы получаем представление нашей категории

В этом подробно обсуждаемом в книге сюжете естественно видеть аналог классического операционного исчисления. Обертывающая алгебра есть одновременно алгебра левоинвариантных дифференциальных операторов на группе Ли, а ее действие в представлениях есть аналог преобразования Фурье полиномиальных дифференциальных операторов.

Интегральные формулы сплетающих операторов основной неунитарной серии вкупе с детальным описанием присоединенного действия алгебры Ли ḱ в $U(\mathfrak{g})$ позволяют описать алгебру $U_{\lambda \lambda} / \operatorname{ker} \pi_{\lambda} U(\mathfrak{g})$ и классифицировать ее неприводимые представления. Этот результат используется затем при классификации неприводимых представлений комплексной полупростой группы Ли.

Если видеть в обсуждаемой работе "спортивную сторону", то теорема ЖелобенкоНаймарка давно превзойдена. С другой стороны, книга остается содержательным текстом по комплексным полупростым группам (которые вообще поняты лучше вещественных) и вместе с сопутствующими статьями может оказаться "базисом" для поступательного движения в будущем. K сожалению, книга написана в стиле, общепринятом в современной математике, - она является юридически точным текстом, рассчитанным на специалистов. ${ }^{20} \mathrm{~B}$ итоге менее известно и менее понято, чем это того бы заслуживало, "операционное исчисление". Важным текстом по этому предмету являются записки М. Дюфло [14] (1974). ${ }^{21}$

В настоящее время в печати находится незавершенная книга Желобенко "Операционное исчисление на комплексных полупростых группах Ли" (мы ее пока не видели). Судя по названию, она продолжает обсуждавшиеся выше сюжеты; хотелось бы надеяться, что она может способствовать вхождению данных вопросов в сотmоn knowledge.

4. Книга "Представления редуктивных алгебр Ли" [55] (1994). Эта работа и связанный с ней цикл статей, возможно, составляют наиболее интересную в настоящее время часть оригинального научного наследия Д. П. Желобенко. Основная часть книги посвящена изложении теории так называемых редукционных алгебр, или алгебр Микельссона [30].

19Эта структура рассматривается также в книге Диксмье [13] (1974, глава 9) со ссылкой на работу Леповского и Макколума [28] (1973), впрочем, структура достаточно естественна и, может быть, где-то вводилась и ранее.

${ }^{20}$ Впоследствии Дмитрий Петрович сожалел, что поддался влиянию “бурбакизма", тогда еще только начинавшего входить в моду.

${ }^{21}$ Под влиянием этих записок техника Желобенко использовалась в работе П. Торассо [35], кроме того, она использовалась в одном из вариантов доказательства гипотезы Баума-Конна у Винсента Лафорга [26]. 
Пусть $\mathfrak{g} \supset \mathfrak{k}$ - комплексные алгебры Ли, причем $\mathfrak{k}$ редуктивна. ${ }^{22}$ Нас интересует задача ограничения конечномерных представлений $\mathfrak{g}$ на $\mathfrak{k}^{23}$ Разложим $\mathfrak{k}$ в сумму понижающей, диагональной (картановской) и повышающей подалгебр: $\mathfrak{k}=\mathfrak{n}_{-}+$ $\mathfrak{h}+\mathfrak{n}_{+}$. Рассмотрим идеал $U(\mathfrak{g}) \mathfrak{n}_{+}$и фактор $U(\mathfrak{g}) / U(\mathfrak{g}) \mathfrak{n}_{+}$по этому идеалу. Редукционная алгебра (или алгебра Микельссона $S(\mathfrak{g}, \mathfrak{k})$ ) определяется как подпространство $\mathfrak{n}_{+}$-старших векторов в $U(\mathfrak{g}) / U(\mathfrak{g}) \mathfrak{n}_{+}$.

Легко убедиться, что $S(\mathfrak{g}, \mathfrak{k})$ в самом деле является алгеброй и что она действует на пространстве $V^{\mathfrak{n}}+$ старших векторов любого $\mathfrak{g}$-модуля $V$. Далее ${ }^{24} \mathfrak{g}$-модуль $V$ восстанавливается из $S(\mathfrak{g}, \mathfrak{k})$-модуля $V^{\mathfrak{n}}+$.

Важнейшим инструментом изучения редукционных алгебр является "экстремальный проектор" Р. М. Ашеровой-Ю. Ф. Смирнова-В. Н. Толстого (1971), [1], [2]. Для алгебры Ли $\mathfrak{s l}_{2}$ со стандартным базисом $e, h$ и $f$ он был получен П. О. Лоудином [29] в 1964 г.:

$$
P=\sum_{n=0}^{\infty} \frac{f^{n} e^{n}}{\prod_{j=1}^{n}(h+j+1)}=\prod_{n=1}^{\infty}\left(1-\frac{f e}{n(h+n+1)}\right) .
$$

Это выражение определяет оператор в любом конечномерном $\mathfrak{s l}_{2}$-модуле ${ }^{25}$, оператор $P$ удовлетворяет соотношениям

$$
e P=P f=0, \quad P^{2}=P .
$$

Иными словами, $P$ есть проектор на подпространство старших векторов.

Р. М. Ашерова, Ю. Ф. Смирнов и В. Н. Толстой нашли аналогичное выражение для произвольной полупростой алгебры k. А именно, для произвольного положительного корня $\alpha$ рассматривается соответствующая подалгебра $\mathfrak{s l}_{2}$ с базисом $e_{\alpha}, h_{\alpha}, f_{\alpha}$, выписывается соответствующий проектор $P_{\alpha}$, а дальше берется произведение $\prod P_{\alpha}$ по всем положительным корням в правильном ${ }^{26}$ порядке.

В формуле для $P$ присутствует деление на элементы обертывающей алгебры $U(\mathfrak{k})$, т. е. сам $P$ в $U(\mathfrak{k})$ не содержится.

В связи с этим Желобенко вводит некоторое расширение алгебры $U(\mathfrak{k})$ (с частично разрешенным делением) и отождествляет его с локально конечными эндоморфизмами некоторого универсального модуля, так что существование и единственность проекционного оператора становятся тавтологией.

Привлечение проектора вынуждает локализовать ${ }^{27}$ алгебру $S(\mathfrak{g}, \mathfrak{k})$ по выражениям вида $h_{\gamma}+k$, где $h_{\gamma}-$ кокорень, а $k$ - целое число. В локализованной алгебре $Z(\mathfrak{g}, \mathfrak{k})$ появляются естественные образующие $z_{g}=P g$, где $g$ пробегает ортогональное дополнение к $\mathfrak{k}$ в $\mathfrak{g}$. Образующие $z_{g}$ удовлетворяют квадратично-линейным соотношениям с коэффициентами, являющимися рациональными выражениями от картановских элементов. Полное или частичное знание этих соотношений позволяет получать различную информацию о представлениях алгебры Ли g. Так, описание алгебры Микельссона для пары $\mathfrak{g l}_{n+1} \supset \mathfrak{g l}_{n}$ позволяет в конце концов получить явные формулы действия образующих $\mathfrak{g l}_{n}$ в базисе Гельфанда-Цетлина [2], а исследование пар, связанных с симметрическими пространствами, применяется в классификации некоторых серий модулей Хариш-Чандры вещественных групп Ли. ${ }^{28}$

\footnotetext{
${ }^{22}$ Стоит обратить внимание на неожиданную степень общности, подалгебра $\mathfrak{k}$ не обязательно симметрическая.

23 Лучше иметь в виду несколько большую общность; рассматриваются модули со старшим весом над $\mathfrak{g}$, чье ограничение на $\mathfrak{k}$ является суммой (не обязательно прямой) модулей со старшим весом.

24 при выполнении условий предыдущей сноски.

${ }^{25} \mathrm{OH}$ определен и в большей общности, но это требует отдельного обсуждения.

26 так называемом нормальном.

27 т. е. частично разрешить деление.

${ }^{28}$ О других приложениях см. [32].
} 
Определяющие свойства образующих $z_{g}$ можно “транспонировать" и получить другие образующие $z_{g}^{\prime}$, также удовлетворяющие квадратично-линейным соотношениям. Желобенко строит оператор $Q$ такой, что $z_{g}^{\prime}=Q g$. Оператор $Q$ (известный специалистам как коцикл Желобенко) также факторизуется по корням и похож на $P$ в случае $\mathrm{sl}_{2}$ :

$$
Q_{\alpha}(x)=\sum_{n=0}^{\infty} \operatorname{Ad}\left(e_{\alpha}\right)^{n} x \cdot f_{\alpha}^{n} \frac{(-1)^{n}}{\prod_{j=1}^{n} j\left(h_{\alpha}-j+1\right)},
$$

где $\operatorname{Ad}\left(e_{\alpha}\right) x:=\left[e_{\alpha}, x\right]$ - оператор присоединенного представления. Позднее операторы (2) появились в математической физике под именем динамической группы Вейля, см. работы Г. Фельдера [16], П. Этингофа и А. Варченко [15], а также [24]. При этом общее понимание самой формулы (2) до сих пор не является удовлетворительным.

Обсуждаемая книга Желобенко содержит довольно много мелких неточностей, кроме того, последняя глава, на наш взгляд, неудачна. Но в целом книга является яркой и очень оригинальной работой.

5. Книга "Основные структуры и методы теории представлений" [56] (2004). Работа очень необычна по стилю и структуре. Это текст “лоскутной структуры", по-видимому, изначально не предназначенный для "систематического изучения" в обычном смысле этого слова. Книга состоит из отрывков, каждый из которых, однако, вполне интересен и пригоден для чтения. Они, с одной стороны, рассчитаны на начинающих, с другой стороны, математик-профессионал может найти в них немало интересного и неожиданного.

6. Неразложимые представления группы Лоренца (1958-1959). Бесконечномерные неунитарные представления группы Лоренца $\operatorname{SL}(2, \mathbb{C})$ (как, впрочем, и полупростых групп вообще) не являются вполне приводимыми (т. е., вообще говоря, не разлагаются в прямую сумму неприводимых представлений). В частности, при исключительных значениях параметров (см. выше) представление основной серии $\mathrm{SL}(2, \mathbb{C})$ расщепляется на конечномерное представление и бесконечномерный остаток ${ }^{29}$. Две заметки Желобенко в "Докладах АН СССР" [37], [38] посвящены задаче описания всех неразложимых представлений группы Лоренца (с конечным композиционным рядом). Сцепляться могут лишь подпредставления, имеющие один и тот же центральный характер.

Желобенко придумал способ свести данную задачу к задаче конечномерной линейной алгебры. А именно, нужно классифицировать наборы линейных операторов $d_{+}: V \rightarrow W, d_{-}: W \rightarrow V, \delta: W \rightarrow W$ таких, что блочные матрицы

$$
a:=\left(\begin{array}{cc}
0 & d_{-} \\
d_{+} & 0
\end{array}\right), \quad b:=\left(\begin{array}{cc}
0 & 0 \\
0 & \delta
\end{array}\right)
$$

удовлетворяют соотношениям:

$$
a b=b a, \quad a, b \text { нильпотентны. }
$$

Эта задача линейной алгебры, в свою очередь, оказалась весьма любопытной, она была решена И. М. Гельфандом и В. А. Пономаревым [19] (1968).

Аналогичная редукция к конечномерной задаче линейной алгебры была проведена для групп $\mathrm{SO}(n, 1)$ и $\mathrm{SU}(n, 1)$, см. [23]. Для произвольной вещественной полупростой группы в [7] был предложен общий метод редукции (далекий от эффективного алгоритма). Вероятно, эти результаты не являются окончательными.

\footnotetext{
29 который сам является точкой основной серии.
} 
7. Базисы Гельфанда-Цетлина. Конечномерные неприводимые представления полупростых групп допускают простую параметризацию (теорема Эли Картана о старшем весе), однако индивидуальные представления устроены сложно и описание их явных реализаций является весьма замысловатой задачей. Подобная проблема еще раньше возникла для симметрических групп $S_{n}$. В начале XX века Альфред Юнг [36] предложил способ построения моделей представлений с помощью последовательного ограничения представления на подгруппы $S_{n} \supset S_{n-1} \supset S_{n-2} \supset \ldots$. Данный способ был применен И. М. Гельфандом и М.Л. Цетлиным [20] (1950) к классическим группам $\mathrm{GL}(n, \mathbb{C})$ и $\mathrm{O}(n, \mathbb{C})$. Напомним, что, ограничивая неприводимое конечномерное представление $\mathrm{GL}(n, \mathbb{C})$ на $\mathrm{GL}(n-1, \mathbb{C})$, мы получаем однократную прямую сумму. Далее мы ограничиваем каждое из полученных представлений на $\operatorname{GL}(n-2, \mathbb{C})$ и т. д. В итоге мы получаем разложение начального пространства в прямую сумму одномерных подпространств. Выбирая в каждом из них по вектору (не канонически), мы получаем базис. Эта часть рассуждений достаточно тривиальна ${ }^{30}$. Однако глубоким результатом являются явные формулы для действия генераторов алгебры Ли $\mathfrak{g l}(n, \mathbb{C})$ в этом базисе. Гельфанд и Цетлин анонсировали эти формулы в двух заметках в "Докладах АН СССР".

Впоследствии разные авторы опубликовали несколько различных доказательств, самые ранние - это работы Желобенко [39] и Г. Бэрда, Л. Биденхарна [3]. Важнее, что Желобенко удалось развить на этом приемы, пригодные для решения других спектральных задач (и это было одной из отправных точек для книг [47], [55], см. также обзор [52]). ${ }^{31}$

8. Голоморфные семейства сплетающих операторов и "операторы дискретной симметрии". Наконец, обсудим результаты Желобенко по сплетающим операторам, частично они включены в книгу "Гармонический анализ на полупростых комплексных группах", но есть и более поздняя статья [51] о вещественных группах.

Выше (п. 3) была описана конструкция сплетающих операторов для представлений основной серии $\mathrm{GL}(n, \mathbb{C})$; для каждой перестановки $\sigma \in S_{n}$ параметров она дает мероморфную операторнозначную функцию от параметров представления ${ }^{32}$ с возможными полюсами и нулями в точках приводимости. Например, для группы $\mathrm{GL}(n, \mathbb{C})$ это функция от $p_{1}, \ldots, p_{n}$. Числа же $p_{j}-q_{j}$ целые, т. е. мы имеем $\mathbb{Z}^{n}$ мероморфных функций.

Поведение таких функций в точках приводимости - важный и сложный вопрос, которому посвящено неисчислимое количество статей. Желобенко (в случае комплексных групп) вводит неочевидную нормировку, делающую это семейство операторов голоморфным и нигде не обращающимся в нуль. В частности, это дает явные формулы для сплетающих операторов и в точках приводимости.

Однако сплетающие операторы для основных серий этим не исчерпываются. Иногда (только в точках приводимости) бывают операторы, связывающие представления с разными наборами $\left\{p_{j}-q_{j}\right\}^{33}$. Желобенко понял, что существование таких исключительных симметрий связано с естественным действием дубля симметрической

${ }^{30}$ Можно сослаться на формулу Пьери.

${ }^{31}$ Отметим также, что Желобенко (1962, см. [39], [47]) получил спектр ограничения конечномерного представления $\operatorname{Sp}(2 n, \mathbb{C})$ на $\operatorname{Sp}(2 n-2, \mathbb{C})$. Он не однократен, однако похож на спектр ограничения $\mathrm{O}(k, \mathbb{C})$ на $\mathrm{O}(k-2, \mathbb{C})$; это заставляло думать, что базисы Гельфанда-Цетлина для $\operatorname{Sp}(2 n, \mathbb{C})$ существуют. Вопрос довольно много обсуждался, см., например, подход, предложенный А. А. Кирилловым и реализованный В. В. Штепиным [34] (1986). Задача в итоге была решена А. И. Молевым [31] (1999). Отметим также, что заметка Желобенко на эту тему в "Успехах" (1987, т. 42, № 6) ошибочна.

32 или, согласно Брюа [9], функцию со значениями в $G$-инвариантных обобщенных функциях на произведении флаговых многообразий.

${ }^{33}$ Кажется, для группы Лоренца это заметил Наймарк [33], разбираясь с вырождением основной серии. 
группы $S_{n} \times S_{n}$ на множестве наборов $p_{1}, \ldots, p_{n}, q_{1}, \ldots, q_{n}$. Он также дал для этих операторов явные конструкции.

Для произвольной вещественной полупростой алгебры Ли $\mathfrak{g}$ это связано с действием группы Вейля комплексификации $\mathfrak{g}_{\mathbb{C}}$ на двойственном пространстве к картановской подалгебре. ${ }^{34}$

Насколько мы помним Москву 1970-80-х годов, Д. П. Желобенко выглядел относительно обособленной фигурой. Но книгу "Компактные группы Ли" читали очень многие, математики и физики, студенты и профессионалы, в Советском Союзе и за границей. Нам еще кажется, что деятельность Желобенко повлияла на становление, интересы и вкусы целого ряда молодых тогда математиков: А. В. Зелевинского, Г. Л. Литвинова, А. И. Молева, М. Л. Назарова, Ю. А. Неретина, Г. И. Ольшанского, В. Н. Толстого, С. М. Хорошкина, И. В. Чередника, ${ }^{35}$ хотя никто из них ни формально, ни фактически не был учеником Желобенко.

Наша статья была написана по совету Р. С. Исмагилова. Нам также помогли М. С. Аль-Натор, Мишель Дюфло, Тамара Ивановна Желобенко, А. В. Зелевинский, Г. Л. Литвинов, А. С. Казаров, М.Л. Назаров, В. Р. Нигматуллин, В. Ф. Молчанов, Г. И. Ольшанский, В. Н. Толстой, Н. Я. Хелемский, В. И. Чилин.

Ю. А. Неретин, С. М. Хорошкин

\section{Список литературы}

[1] Р. М. Ашерова, Ю.Ф. Смирнов, В.Н. Толстой, "Проекционные операторы для полупростых алгебр Ли", ТМФ, 8:2 (1971), 255-271; англ. пер.: R. M. Asherova, Yu. F. Smirnov, V. N. Tolstoi, "Projection operators for simple Lie groups", Theoret. and Math. Phys., 8:2 (1971), 813-825.

[2] Р. М. Ашерова, Ю.Ф. Смирнов, В.Н. Толстой, “Проекционные операторы для полупростых алгебр Ли. II. Общая схема построения понижающих операторов. Случай групп $S U(n) ", \quad T M \Phi, \mathbf{1 5 : 1}$ (1973), 107-119; англ. пер.: R. M. Asherova, Yu. F. Smirnov, B. N. Tolstoi, "Projection operators for simple Lie groups. II. General scheme for constructing lowering operators. The groups $S U(n)$ ", Theoret. and Math. Phys., 15:1 (1973), 392-401.

[3] G. E. Baird, L. C. Biedenharn, "On representations of the semisimple Lie groups. II", J. Math. Phys., 4 (1963), 1449-1466.

[4] A. Beilinson, J. Bernstein, "Localisation de g-modules", C. R. Acad. Sci. Paris Sér. I Math., 292:1 (1981), 15-18.

[5] Ф. А. Березин, "Операторы Лапласа на полупростых группах", Докл. АН СССР, 107 (1956), 9-12.

[6] Ф. А. Березин, "Операторы Лапласа на полупростых группах", Tp. MMO, 6, 1957, 371-463.

[7] J. Bernstein, I. Gelfand, S. Gelfand, "Structure locale de la catégorie des modules de Harish-Chandra", C. R. Acad. Sci. Paris Sér. A-B, 286:11 (1978), A495-A497.

[8] A. Borel, N.R. Wallach, Continuous cohomologies, discrete subgroups, and representations of reductive groups, Ann. of Math. Stud., 94, Princeton University Press, Princeton, NJ; University of Tokyo Press, Tokyo, 1980.

[9] F. Bruhat, "Sur les représentations induites des groupes de Lie", Bull. Soc. Math. France, 84 (1956), 97-205.

\footnotetext{
34Эвристическое объяснение для читателя, немного знакомого с предметом: собственные числа операторов Лапласа постоянны на таких орбитах.

${ }^{35}$ Разумеется, в отношении себя авторы это подтверждают.
} 
[10] J.-L. Brylinski, M. Kashiwara, "Kazhdan-Lusztig conjecture and holonomic systems", Invent. Math., 64:3 (1981), 387-410.

[11] W. Casselman, "The differential equations satisfied by matrix coefficients", Unpublished manuscript, 1975.

[12] W. Casselman, D. Miličić, "Asymptotic behavior of matrix coefficients of admissible representations", Duke Math. J., 49:4 (1982), 869-930.

[13] Ж. Диксмье, Универсальные обертъьвающие алгебры, Мир, М., 1978; пер. c франц.: J. Dixmier, Algèbres enveloppantes, Cahiers Scientifiques, 37, Gauthier-Villars, Paris-Brussels-Montreal, QC, 1974.

[14] M. Duflo, "Représentations irréductibles des groupes semi-simples complexes", Analyse harmonique sur les groupes de Lie (Nancy-Strasbourg, 1973-75), Lecture Notes in Math., 497, Springer, Berlin, 1975, 26-88.

[15] P. Etingof, A. Varchenko, "Dynamical Weyl groups and applications", Adv. Math., 167:1 (2002), 74-127.

[16] G. Felder, "Conformal field theory and integrable systems associated with elliptic curves", Proceedings of the International Congress of Mathematicians, Vols. 1, 2 (Zürich, 1994), Birkhäuser, Basel, 1995, 1247-1255.

[17] M. Flensted-Jensen, "Discrete series for semisimple symmetric spaces", Ann. of Math. (2), 111:2 (1980), 253-311.

[18] И. М. Гельфанд, М.А. Наймарк, "Унитарные представления классических групп", Тр. МИАН, 36, Изд-во АН СССР, М.-Л., 1950.

[19] И. М. Гельфанд, В.А. Пономарев, "Неразложимые представления группы Лоренца”, УМH, 23:2 (1968), 3-59; англ. пер.: I. M. Gel'fand, V. A. Ponomarev, "Indecomposable representations of the Lorentz group", Russian Math. Surveys, 23:2 (1968), 1-58.

[20] И. М. Гельфанд, М. Л. Цетлин, "Конечномерные представления группы унимодулярных матриц", Докл. АН СССР, 71:5 (1950), 825-828.

[21] R. Godement, "Theory of spherical functions. I", Trans. Amer. Math. Soc., 73:3 (1952), 496-556.

[22] Harish-Chandra, "Representations of semi-simple Lie groups. II", Trans. Amer. Math. Soc., 76 (1954), 26-65.

[23] С. М. Хорошкин, "Неразложимые представления групп Лоренца", Функи. анализ и его прил., 15:2 (1981), 50-60; англ. пер.: S. M. Khoroshkin, "Irreducible representations of Lorentz groups", Funct. Anal. Appl., 15:2 (1981), 114-122.

[24] S. Khoroshkin, M. Nazarov, "Yangians and Mickelsson algebras. I", Transform. Groups, 11:4 (2006), 625-658; arXiv: math/0606265.

[25] A.W. Knapp, Representation theory of semi-simple groups. An overview based on examples, Princeton Math. Ser., 36, Princeton University Press, Princeton, NJ, 1986.

[26] V. Lafforgue, K-théory bivariante pour les algèbres de Banach et conjecture de Baum-Connes, Thèse de doctorat, Université Paris-Sud, Paris, 1999.

[27] R. P. Langlands, "On classification of irreducible representations of real algebraic groups", Unpublished Manuscript, 1973.

[28] J. Lepowsky, G. W. McCollum, "On determination of irreducible modules by restriction to a subalgebra", Trans. Amer. Math. Soc., 176 (1973), 45-57.

[29] P.-O. Löwdin, "Angular momentum wavefunctions constructed by projector operators", Rev. Modern Phys., 36 (1964), 966-976.

[30] J. Mickelsson, "Step algebras of semi-simple subalgebras of Lie algebras", Rep. Mathematical Phys., 4:4 (1973), 307-318. 
[31] A.I. Molev, "A basis for representations of symplectic Lie algebras", Comm. Math. Phys., 201:3 (1999), 591-618.

[32] A.I. Molev, "Yangians and transvector algebras", Discrete Math., 246:1-3 (2002), $231-253$.

[33] М.А. Наймарк, Линейные представления группы Лорениа, Физматгиз, М., 1958; англ. пер.: M. A. Naimark, Linear representations of the Lorentz group, The Macmillan, New York, 1964.

[34] В. В. Штепин, "Разделение кратных точек спектра при редукции $\operatorname{sp}(2 n) \downarrow \operatorname{sp}(2 n-$ 2)", Функи. анализ и его прил., 20:4 (1986), 93-95; англ. пер.: V. V. Shtepin, "Separation of multiple points of spectrum in the reduction $\operatorname{sp}(2 n) \downarrow \operatorname{sp}(2 n-2)$ ", Functional Anal. Appl., 20:4 (1986), 336-338.

[35] P. Torasso, "Le théorème de Paley-Wiener pour l'espace des fonctions indéfinitement différentiables et à support compact sur un espace symétrique de type non-compact", J. Funct. Anal., 26:2 (1977), 201-213.

[36] A. Young, "On quantitative substitutional analysis. VI", Proc. London Math. Soc. (2), 31:2 (1931), 253-288.

[37] Д. П. Желобенко, "Описание некоторого класса линейных представлений группы Лоренца", Докл. АН СССР, 121:4 (1958), 585-589.

[38] Д. П. Желобенко, “Линейные представления группы Лоренца”, Докл. АН CCCP, 126:5 (1959), 935-938.

[39] Д. П. Желобенко, "Классические группы. Спектральный анализ конечномерных представлений", УМH, 17:1 (1962), 27-120; англ. пер.: D. P. Zhelobenko, "The classical groups. Spectral analysis of their finite-dimensional representations", Russ. Math. Surveys, 17:1 (1962), 1-94.

[40] Д. П. Желобенко, "К теории линейных представлений комплексных и вещественных групп Ли", Тр. ММO, 12 (1963), 53-98.

[41] Д. П. Желобенко, "Гармонический анализ на полупростых группах Ли. I", Изв. АН СССР. Сер. матем., 27:6 (1963), 1343-1394.

[42] Д. П. Желобенко, "Симметрия в классе элементарных представлений полупростой комплексной группы Ли", Функи. анализ и его прил., 1:2 (1967), 15-38; англ. пер.: D.P. Zhelobenko, "Symmetry in a class of elementary representations of a semisimple complex Lie group", Funct. Anal. Appl., 1:2 (1967), 103-121.

[43] Д. П. Желобенко, "Анализ неприводимости в классе элементарных представлений полупростой комплексной группы Ли”, Изв. АН СССР. Сер. матем., 32:1 (1968), 108-133; англ. пер.: D.P. Želobenko, "The analysis of irreducibility in the class of elementary representations of a complex semisimple Lie group", Math. USSR-Izv., 2:1 (1968), 105-128.

[44] Д. П. Желобенко, "Операционное исчисление на полупростой комплексной группе Ли", Изв. АН СССР. Сер. матем., 33:5 (1969), 931-973; англ. пер.: D. P. Želobenko, "Operational calculus on a complex semisimple Lie group", Math. USSR-Izv., 3:5 (1969), 881-916.

[45] Д.П. Желобенко, "О гармоническом анализе функций на полупростых группах Ли. II”, Изв. АН СССР. Сер. матем., 33:6 (1969), 1255-1295; англ. пер.: D. P. Želobenko, "Harmonic analysis of functions on semisimple Lie groups. II", Math. USSR-Izv., 3:6 (1969), 1183-1217.

[46] Д.П. Желобенко, "О неприводимых представлениях класса 0 полупростой комплексной группы Ли”, Функи. анализ и его прил., 4:2 (1970), 85-86; англ. пер.: D. P. Zhelobenko, "On the irreducible representations of a complex semisimple Lie group", Funct. Anal. Appl., 4:2 (1970), 163-165. 
[47] Д. П. Желобенко, Компактные группы Ли и их представления, Наука, М., 1970; англ. пер.: D. P. Želobenko, Compact Lie groups and their representations, Transl. Math. Monogr., 40, Amer. Math. Soc., Providence, RI, 1973.

[48] Д. П. Желобенко, "Классификация экстремально неприводимых и нормально неприводимых представлений полупростой комплексной связной группы Ли", Изв. АН СССР. Сер. матем., 35:3 (1971), 573-599; англ. пер.: D. P. Želobenko, "Classification of extremally irreducible and normally irreducible representations of semisimple complex connected Lie groups", Math. USSR-Izv., 5:3 (1971), 589-613.

[49] Д. П. Желобенко, "Циклические модули для комплексных полупростых групп Ли", Изв. АН СССР. Сер. матем., 37:3 (1973), 502-515; англ. пер.: D. P. Želobenko, "Cyclic modules for a complex semisimple Lie group", Math. USSR-Izv., 7:3 (1973), 597-510.

[50] Д. П. Желобенко, Гармонический анализ на полупростых комплексных группах Ли, Соврем. пробл. матем., Наука, М., 1974.

[51] Д. П. Желобенко, “Операторы дискретной симметрии для редуктивных групп Ли”, Изв. АН СССР. Сер. матем., 40:5 (1976), 1055-1083; англ. пер.: D. P. Želobenko, "Discrete symmetry operators for reductive Lie groups", Math. USSR-Izv., 10:5 (1976), 1003-1029.

[52] D. P. Zhelobenko, "On Gelfand-Zetlin bases for classical Lie algebras", Representations of Lie groups and Lie algebras (Budapest, 1971), Akad. Kiadó, Budapest, 1985, 79-106.

[53] Д. П. Желобенко, “Аналог базиса Гельфанда-Цетлина для симплектических алгебр Ли", УМН, 42:6 (1987), 193-194; англ. пер.: D. P. Zhelobenko, "An analogue of the Gel'fand-Tsetlin basis for symplectic Lie algebras", Russian Math. Surveys, 42:6 (1987), 247-248.

[54] D. P. Zhelobenko, "An introduction to the theory of $S$-algebras over reductive Lie algebras", Representations of Lie groups and related topics, Adv. Stud. Contemp. Math., 7, Gordon and Breach, New York, 1990, 155-221.

[55] Д. П. Желобенко, Представления редуктивных алгебр Ли, Наука, М., 1994.

[56] Д.П. Желобенко, Основные структуры и методы теории представлений, МЦНМО, М., 2004.

[57] Д. П. Желобенко, Операчионное исчисление на комплексных полупростых групnax Ли, МЦНМО (в печати).

[58] Д. П. Желобенко, Гауссовы алгебры, МЦНМО (в печати).

[59] Д. П. Желобенко, М. А. Наймарк, “Описание вполне неприводимых представлений полупростой комплексной группы Ли”, Докл. АН CCCP, 171:1 (1966), 25-28; англ. пер.: D. P. Želobenko, M. A. Naǐmark, "Description of completely irreducible representations of a semi-simple complex Lie group", Soviet Math. Dokl., 7 (1966), 1403-1406.

[60] Д. П. Желобенко, М. А. Наймарк, "Описание вполне неприводимых представлений полупростой комплексной группы Ли”, Изв. АН СССР. Сер. матем., 34:1 (1970), 57-82; англ. пер.: D. P. Želobenko, M. A. Naĭmark, "Description of the completely irreducible representations of a complex semisimple Lie group", Math. USSR-Izv., 4:1 (1970), 59-83. 